\title{
Langue, parole et discours: dédoublement guillaumien de la distinction saussurienne
}

\author{
Anne-Gaëlle Toutain ${ }^{1, *}$ \\ ${ }^{1}$ Université de Berne - Laboratoire « Histoire des théories linguistiques » (UMR7597)
}

\begin{abstract}
Résumé. Cet article propose une analyse de l'élaboration guillaumienne de la distinction saussurienne entre langue et parole, en termes de distinction double : entre langue et parole et entre langue et discours. Nous montrons que la singularité de cette élaboration consiste en l'adjonction d'une construction spéculative à la démarche structuraliste d'analyse du donné idiomologique et de la parole. Apparait ainsi en pleine lumière l'empirisme de la problématique guillaumienne. La distinction entre langue et discours constitue le cadre de construction du système linguistique, qui doit permettre de rendre compte de la multiplicité des effets de discours. La distinction entre langue et parole constitue la langue comme principe d'analyse. La construction guillaumienne vise cependant également à rendre compte du mécanisme de la parole. En tant que telle, elle est néanmoins nécessairement spéculative, dans la mesure où elle s'inscrit dans la double problématique structuraliste et des rapports son/sens : la définition de la langue intervient sur le fond de l'acceptation préalable du donné du langage. À la définition saussurienne de la langue comme fonctionnement, ainsi distinguée du langage, répond une dynamisation de la distinction entre langue et parole, dont le langage fournit le cadre: l'objectalité de la langue n'est pas remise en cause, mais construite comme le produit d'un processus langagier.
\end{abstract}

\begin{abstract}
Language (langue), speech (parole) and discourse : Guillaumian duplication of Saussurean distinction. This paper provides an analysis of the Guillaumian elaboration of Saussurean distinction between langue and parole, which results in two distinctions : between language and speech and between language and discourse. We show that the singularity of this elaboration consists in the addition of a speculative construction to the structuralist analysis of idiomological data and speech. The empiricism of the Guillaumian problematics thus appears in full light. The distinction between language and discourse constitutes the framework for building the linguistic system, which must make it possible to account for the multiplicity of discourse effects. The distinction between language and speech constitutes language as a principle of analysis. However, the Guillaumian construction also aims to reflect the mechanism of speech. As such, it is nevertheless necessarily speculative, insofar as it falls within the structuralist problematics and the sound/sense relationships problematics : the definition of langue is based on the prior acceptance of langage as given. To the Saussurian definition of langue as a functioning, thus distinguished from langage, responds a dynamization of the distinction between langue and parole, of which langage provides the
\end{abstract}

\footnotetext{
*Corresponding author : annegaelletoutain@yahoo.fr
} 
framework : the objectal character of language is not called into question, but constructed as the product of a language process.

L'émergence des linguistiques de l'énonciation et des linguistiques cognitives est solidaire d'un dépassement ou d'un outrepassement de la problématique structuraliste. Cette émergence est en effet déterminée par l'identification d'un nouvel objet - la parole, l'énoncé, l'énonciation, le discours, le sujet parlant, ou le fonctionnement cognitif induit ou requis par le langage -, requérant un autre type d'analyse que celle qu'avait mise en œuvre le structuralisme. En tant que telle, elle implique notamment une (ré)élaboration de la distinction saussurienne entre langue et parole, qui se voit, selon le cas, " dépassée », ou repensée sur le modèle chomskyen. Or, d'une part, il existe une radicale différence de problématique entre Saussure et le structuralisme ${ }^{1}$. D'autre part, la distinction saussurienne entre langue et parole est l'instrument d'une rupture épistémologique d'une importance extrême pour la linguistique : avec la conception objectale ${ }^{2}$ de la langue, au profit de la construction d'une extériorité non objectale ${ }^{3}$. À ce double égard, il m'a paru intéressant de soumettre à une analyse épistémologique la lecture guillaumienne de la distinction saussurienne entre langue et parole. La problématique énonciative n'est en effet pas absente de la linguistique de Gustave Guillaume (1883-1960) ${ }^{4}$, non plus que les préoccupations d'ordre cognitiviste ${ }^{5}$. En outre, la linguistique guillaumienne se situe en marge du structuralisme, tout en partageant avec ce dernier plusieurs caractéristiques fondamentales, au premier rang desquelles l'absence de rupture avec la conception objectale de la langue.

Après une rapide présentation des enjeux de la distinction saussurienne entre langue et parole (1), nous verrons que l'élaboration guillaumienne de celle-ci, qui la dédouble (2), adjoint à la démarche structuraliste d'analyse de la parole une construction spéculative (3) ${ }^{6}$. À la définition saussurienne de la langue comme fonctionnement, ainsi distinguée du langage, répond une dynamisation de la distinction, dont le langage fournit le cadre : l'objectalité de la langue n'est pas remise en cause, mais construite comme le produit d'un processus langagier.

\section{Enjeux de la distinction saussurienne entre langue et parole}

La distinction entre langue et parole est à la fois première et relativement tardive dans l'élaboration saussurienne. Dans l'entretien avec Gautier du 6 mai 1911, Saussure la présente comme la «première vérité » (Godel, 1957 : p. 30), et elle est de fait inaugurale, dans la mesure où elle constitue l'objet de la linguistique - la langue - en l'isolant dans le tout du langage, qui est pour sa part un « terrain complexe, multiforme, hétéroclite dans ses différents aspects » (Saussure \& Constantin, 2005 : p. 214). Cette distinction fournit ainsi un cadre de théorisation, et en particulier, elle permet la théorisation de la parole dans le cadre du concept de langue. Contrairement à ce qui est communément affirmé, la distinction saussurienne entre langue et parole n'implique aucune exclusion mais, ce qui est tout différent, une ordonnance du langage à partir et dans le cadre du concept de langue. Distincte du langage, la langue est définie par deux aspects : l'union du son et du sens, corrélative d'un caractère purement psychique, et le caractère social. On lit notamment dans le troisième cours ${ }^{7}$ :

«Quand on a séparé la langue de la faculté du langage, on a séparé :

$1^{\circ}$ ) ce qui est social de ce qui est individuel, $2^{\circ}$ ) ce qui est essentiel de ce qui est plus ou moins accidentel. En effet, on verra plus loin que c'est l'union de l'idée avec un signe vocal qui suffit à constituer toute la langue. » (Saussure \& Constantin, $2005:$ p. 87). 
Ces deux aspects sont solidaires. Le trait distinctif de l'élaboration saussurienne est la théorisation du rapport son/sens, dans le cadre du concept de valeur. Cette « union de l'idée avec un signe vocal » est en effet définie comme «articulation» ou «délimitationcombinaison ", c'est-à-dire comme délimitation produite par la combinaison, combinaison qui est en retour inséparable de cette délimitation, avec laquelle, en réalité, elle se confond. La langue saussurienne n'est pas un ensemble de signes, ni une structure d'appariement du son et du sens, mais elle est découpage d'unités dans le cadre d'un "accouplement» (Saussure, 1997 : p. 22) entre pensée et phonie, un fonctionnement dont son, sens et signe, en tant que linguistiques, et avec eux les idiomes, sont les effets. Il s'agit de ce « fait <en quelque sorte $>$ mystérieux que la pensée-son implique des divisions qui sont les unités finales de la linguistique » (Saussure, $1997:$ p. 21). La langue saussurienne se trouve ainsi définie comme fonctionnement, et non comme objet. Elle est en tant que telle dotée d'un autre type d'extériorité que l'extériorité objectale, extériorité que lui confère son caractère social : ce fonctionnement qu'est la langue, individuel - intérieur à l'individu - par définition, puisqu'il est «articulation de la pensée dans la matière phonique ${ }^{8}$ », est néanmoins socialement codé, caractère social qui en constitue l'extériorité, le mode d'existence (ce que Saussure, dans le deuxième cours, appelle la « vie sémiologique »).

La distinction saussurienne entre langue et parole apparaît ainsi solidaire d'une rupture avec la conception de la langue comme une entité objectale, telle par exemple l'instrument de communication. Elle est en outre corrélative d'une rupture avec la représentation de la langue comme une entité en évolution. Comme indiqué ci-dessus, bien qu'inaugurale, la distinction entre langue et parole apparaît de manière relativement tardive dans l'élaboration saussurienne : dans les cours de linguistique générale (1907-1911) ${ }^{9}$, tandis que le concept de valeur apparaît dès ce qui est sans doute le premier texte de linguistique générale de Saussure, dont la rédaction remonte au début des années 1890 (1891 sqq.), «De l'essence double du langage ». Ce concept émerge en effet dans le cadre de l'élaboration d'une autre distinction saussurienne, qui est pour sa part, sinon inaugurale, théoriquement, $\mathrm{du}$ moins première, historiquement : entre synchronie et diachronie ${ }^{10}$. Saussure établit dans ses premiers textes une équivalence entre phonétique et diachronie d'une part, morphologie et synchronie, d'autre part, équivalence corrélative d'une définition purement synchronique du signe, et d'une opposition entre son-idée - le signe, au sens saussurien - et son, phonologique (non linguistique) ou phonétique (linguistique, diachronique) ${ }^{11}$. Si le signe est délimitation-combinaison, et non entité, alors, en effet, il ne saurait exister dans le temps. On lira ainsi dans la note « Sémiologie » (?1908-1912) :

\begin{abstract}
«Par le fait qu'aucun élément n'existe (ou par mille autres raisons, car nous ne prétendons pas faire une sorte de système cartésien de choses qui tombent sous le sens de tous les côtés), on voit qu'aucun élément n'est (à plus forte raison) en état de se transformer; mais qu'il peut seulement arriver qu'ON lui substitue autre chose, même quand il s'agit de "phonétique", et que par là, toute opération généralement, et toute la différence des opérations réside dans la nature des substitutions auxquelles nous nous livrons en parlant. Qui dit substitution, commence par supposer que le terme auquel on donne un substitut a une existence.... etc. » (Saussure 2002 : p. 266).
\end{abstract}

La notion de transformation se voit ainsi abandonnée par Saussure au profit de celle de substitution, qui renvoie à la délimitation-combinaison : l'élément ne se transforme pas, car il n'existe pas, mais est chaque fois à nouveau, produit de la délimitation-combinaison définitoire de la langue et à laquelle, de ce fait même, "nous nous livrons en parlant ", délimitation-combinaison dont l'effet est ainsi une substitution (c'est chaque fois un nouveau signifiant ou un nouveau signifié, signifiant et signifié dont l'existence est ponctuelle par définition : elle se réduit à l'instant de leur délimitation-combinaison). Dans cette perspective, il ne saurait y avoir aucune continuité entre synchronie et diachronie, ce 
que Saussure affirme de manière extrêmement radicale en parlant de deux objets distincts, sans rapport l'un avec l'autre, par exemple dans « Status et motus » (?1894-1897) :

\begin{abstract}
«Il vaut mieux préciser d'emblée Nous ne considérons pas la linguistique comme une science dans laquelle il y a un bon principe de division à ehercher trouver, mais - à part une ou deux réserves comme une science qui essaie d'assembler en un seul tout deux objets complètement disparates depuis le principe, en se persuadant qu'ils forment un seul objet: Le plus grave est que notre science se trouve satisfaite de cette association, ne paraît point tourmentée du vague sentiment qu'il y a quelque chose de faux dans sa base; ne manifeste aucun malaise devant les conceptions les plus obliques qu'elle accepte chaque jour; se sent même tellement en possession de son objet qu'elle n'éprouve aucune difficulté à extraire de temps en temps de ce désordre général des idées des théories du langage, présentées avec une entière candeur. » (Saussure 2002 : p. 226).
\end{abstract}

La distinction entre synchronie et diachronie, corrélative du concept de valeur, est donc l'instrument d'une rupture avec la représentation de la langue en termes d'entité en évolution, corrélative de la rupture avec la conception objectale que nous venons de voir, dont elle n'est qu'un autre aspect. Or, dans le troisième cours, la distinction entre synchronie et diachronie se trouve ordonnée à la distinction entre langue et parole : elle constitue le «second carrefour» (Saussure \& Constantin, 2005: p. 270), le premier carrefour étant : « doit-on étudier la langue ou la parole ? » (Saussure \& Constantin, 2005 : p. 270), et s'opposent ainsi parole, d'une part, «langue statique » et «langue évolutive » d'autre part. Saussure précise alors :

«C'est le lieu d'ajouter, puisque dans le premier carrefour il s'agissait du choix entre langue et parole, que tout ce qui est diachronique dans la langue naît par la parole. Le rudiment de tout changement dans la langue n'y arrive que par la parole. » (Saussure \& Constantin, 2005 : p. 270).

À la question «Quelle doit être la forme de la linguistique ? (Saussure \& Constantin, 2005 : p. 270), il répond ainsi :

«Toute évolution, tout fait évolutif dans la langue commence par un fait de parole. Il est entendu que ceci reste en dehors du fil des études relatives à la langue. La cause des faits évolutifs de langue gît dans les faits de parole.

Dans les différentes sphères à distinguer on constate qu'il y a des faits homologues qui se répondent de l'une à l'autre.

Mais il ne faut pas pour cela confondre les sphères elles-mêmes. » (Saussure \& Constantin, 2005 : p. 270).

La distinction entre langue et parole constitue ainsi le cadre d'une théorisation de la langue et d'une ordonnance du langage, nouée sur l'idiome dont il s'agit de rendre raison ${ }^{12}$. Dans cette ordonnance, de la même manière que la distinction entre synchronie et diachronie est une autre forme du concept de valeur, elle apparaît comme une autre forme de la distinction entre synchronie et diachronie, qu'elle vient en quelque sorte pluraliser, en séparant ce qui concerne l'étude de l'idiome, fût-ce avec un horizon de linguistique générale, et ce qui concerne les causes et le mécanisme du changement, que Saussure assigne à l'individu. À la rupture avec la conception objectale de la langue (entité objectale, entité en évolution) répond une pluralisation du langage et l'ouverture d'un espace de théorisation.

C'est dans une tout autre problématique que s'élabore la linguistique guillaumienne. 


\section{Langue, parole et discours : dédoublement de la distinction}

Lors de la séance du 20 février 1948 des leçons de linguistique intitulées " Grammaire particulière du français et grammaire générale » de l'année 1947-1948 ${ }^{13}$, Guillaume s'attache à une relecture de la distinction saussurienne entre langue et parole. Il affirme tout d'abord que l'équation "langage = langue + parole » doit être interprétée "selon une relation qui fait du langage le tout, l'intégrale d'une successivité, celle de la langue à la parole - de la langue, présente en nous, en permanence, à l'état de puissance, et de la parole, présente en nous, par moment, à l'état d'effet » (Guillaume, 1988 : p. 110). Cette équation, poursuit-il, a le défaut de ne pas tenir compte du facteur temps :

«Un facteur, dont la formule saussurienne ne tient pas compte, et dont en toute question linguistique il y a lieu de tenir le compte le plus étroit, c'est le facteur temps. Le langage comme tout, comme intégrale, enveloppe une successivité : celle du passage de la langue, présente, dans le sujet parlant, en permanence, en dehors de toute momentanéité par conséquent, à la parole, présente en lui par momentanéité seulement - par momentanéité plus ou moins espacée. » (Guillaume, 1988 : p. 111).

En outre, selon Guillaume, il faut définir ce passage de la langue à la parole un peu différemment de ce que laisse transparaître l'équation saussurienne, dans la mesure où il s'agit moins d'un passage de la langue à la parole que de celui d'une parole virtuelle à une parole actuelle :

« Le sujet parlant trouve la langue en lui prête à servir, à disposition, et il s'en sert pour parler. Il passe, il transite de la langue à la parole. Or, ici, la théorie exposée rencontre un obstacle. Le sujet parlant, dans le moment de l'expression, passe bien, en effet, de la langue à la parole, c'est-à-dire de la langue à la parole effective, momentanée, celle qui s'entend, qui a une existence physique. Mais cette transition de la langue à la parole n'est, en réalité, sans que Saussure en ait fait l'observation, que celle de la parole virtuelle, indissolublement liée au psychisme de la langue, à la parole actuelle, effective et physique. La parole virtuelle, liée à la langue, et faisant partie intégrante de celle-ci, est une parole non physique, silencieuse, que le psychisme des unités de langue apporte avec soi. De la réalité de cette parole non physique il est aisé de se $<$ rendre $>$ compte. Chaque notion de langue emporte avec soi l'idée du ou des sons signifiants, mais l'idée seulement de ce ou de ces sons, pas leur réalité. » (Guillaume, 1988 : p. 112-113).

La parole se voit ainsi redéfinie comme signifiant ${ }^{14}$, et à la langue et à la parole s'adjoint dès lors le discours, qui est, au lieu de la parole, le terme de la successivité constitutive du langage :

«La successivité qu'intègre le langage est, selon la donnée de ce schème d'analyse, celle de la langue au discours, et dans la langue comme dans le discours, il y a liaison et congruence d'un fait de parole et d'un fait de pensée. » (Guillaume, 1988 : p. 113).

La distinction saussurienne se trouve ainsi dédoublée en deux distinctions: langue/discours et pensée/parole ${ }^{15}$. L'année précédente, Guillaume insistait de même sur l'importance de la temporalité, parlant de «linguistique de position ${ }^{16}$. C'est cependant, dans les leçons de cette année-là, une autre insuffisance de la distinction saussurienne qu'il mettait en avant :

«La signification que j'attribue aux quatre termes précités - langue, langage, discours, parole - est très voisine de celle que leur attribue Ferdinand de Saussure dans son Cours de linguistique générale. Elle 
me paraît toutefois comporter une plus grande précision, et résulter d'une appréciation plus exacte de ce qui se passe chez le sujet parlant dans le moment où il parle. » (Guillaume, 1989 : p. 9).

C'est donc le mécanisme de la parole que Guillaume se donne pour objet dans sa construction de la distinction langue/discours. Ainsi ajoute-t-il :

« La distinction que nous avons faite la dernière fois du plan de puissance, où siège en permanence la langue, et du plan de l'effet où se produit momentanément le discours, est une distinction qui aide considérablement à bien saisir ce qu'est en réalité le mécanisme linguistique. » (Guillaume, 1989 : p. 9).

Cet objet, ainsi que la dynamisation de la distinction saussurienne qui en est corrélative, me paraissent constituer la singularité de la relecture guillaumienne de cette distinction. Comme nous allons le voir dans ce qui suit, cette relecture s'inscrit néanmoins dans le paradigme structuraliste.

Il apparaît d'emblée, en considération de la redéfinition guillaumienne du langage comme "intégrale d'une successivité », qu'il ne s'agit pas, comme chez Saussure, du découpage d'un objet spécifiquement linguistique dans le tout du langage, en lui-même inconnaissable, mais, comme chez Jakobson ${ }^{17}$ - et comme chez les structuralistes en général - d'un partage d'objet. La reformulation de l'équation saussurienne en termes d'intégrale - avec le symbole mathématique correspondant - appelle ainsi, dans la leçon du 20 février 1947, le commentaire suivant : "La langue est bien, comme l'a indiqué F. de Saussure, un tout relatif à deux composantes : la langue et la parole. » (Guillaume, 1988 : p. 111). Le langage est ainsi pris dans son évidence d'objet donné, et ne fait l'objet d'aucune définition - comme nous le verrons ci-dessous, il n'en va pas tout à fait de même de la langue. Corrélativement, l'élaboration guillaumienne s'inscrit dans la double problématique structurale et des rapports son/sens qui est définitoire du structuralisme européen. On lit ainsi notamment dans la leçon du 29 novembre 1946 :

« Des deux côtés, dans la vue de faciliter l'obtention de la congruence parfaite recherchée vainement et inlassablement, il y a construction de systèmes. Le système qui se construit du côté de la parole est le système phonologique. Celui qui se construit du côté de la pensée est le système psychique.

Une langue suppose un accord, merveilleusement réalisé, après des millénaires de tentatives, entre les deux systèmes institués : le système phonologique d'un côté, le système psychique de l'autre. Ce qu'on appelle le génie d'une langue, c'est cet accord, son degré de réussite. Il est répété, comme un principe à ne jamais perdre de vue, que le fait linguistique est essentiellement la découverte et le maintien d'un accord, toujours insuffisant, entre les deux systèmes: l'un extérieur, phonologique - et plus généralement sémiologique -, l'autre intérieur, psychique. » (Guillaume, 1989 : p. 5).

Si la parole est signifiant, le rapport entre signifiant et signifié (pensée) est conçue en termes d'accord ou de congruence, donc en termes de rapport son/sens. À cette représentation répond une hypothèse structurale : la langue est constructrice de systèmes. On notera, comme remarquable, l'expression « fait linguistique », que Guillaume n'a très probablement pas empruntée à Saussure, mais que l'on trouve chez Saussure, dans le troisième cours :

«Mais il n'y a rien du tout de distinct dans la pensée avant le signe linguistique. Ceci est le principal. D'un autre côté, il vaut aussi la peine de se demander si en face de ce royaume des idées tout à fait 
confus le royaume du son offrirait d'avance des unités bien distinctes (pris en lui-même en dehors de l'idée).

Il n'y a pas non plus dans le son des unités bien distinctes, circonscrites d'avance. C'est entre deux que le fait linguistique se passe.

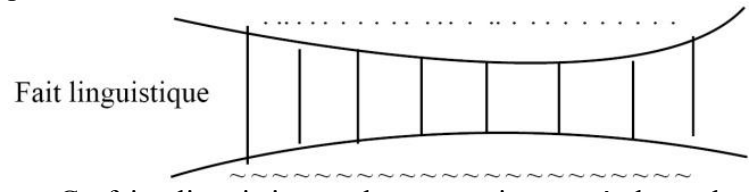

Ce fait $<$ linguistique $>$ donnera naissance à des valeurs qui elles $<$ pour la première fois $>$ seront déterminées, mais qui n'en resteront pas moins des valeurs, avec le sens qu'on peut attacher à ce mot. (Saussure \& Constantin, 2005 : p. 285).

Ce « fait linguistique » - la délimitation-combinaison - a donc valeur théorisante chez Saussure, puisqu'il rend compte de l'existence des signes - dès lors définis comme des valeurs. Guillaume désigne en revanche par ce syntagme le rapport son/sens constitutif de la définition traditionnelle de la langue, qui n'a aucune valeur théorisante puisqu'il est ce que « suppose » - et non institue - la langue. Dans la leçon du 6 décembre 1946, Guillaume parle d'inséparabilité :

« Pendant l'acte de langage, la parole est effective. Elle cesse au discours produit. Elle n'existe pas encore effectivement avant l'acte de langage, c'est-à-dire dans la langue, sur le plan de puissance. Le plan de puissance est un plan où la parole est, conformément à la nature du plan occupé, de la parole en puissance. À chaque notion, prise dans la langue, en dehors de tout acte de langage, s'attache inséparablement le souvenir d'un fragment de parole, souvenir qui fait en quelque sorte corps avec elle. Ce fragment de parole, dont le souvenir, l'idée, fait corps avec la notion, c'est le signifiant. La notion que le signifiant emporte avec lui, à laquelle il s'attache inséparablement, c'est le signifié.

On se trouve en présence d'une symphyse, d'une soudure psychique remarquable, selon laquelle un fragment de parole appelle à soi, automatiquement, un fragment de pensée, cependant que le fragment de pensée, réciproquement, appelle le fragment de parole. » (Guillaume, 1989 : p. 13).

Il s'agit là, cependant - comme chez Damourette et Pichon -, d'une description de ce « fait linguistique », alors que celui-ci requerrait théorisation. Notons à cet égard, dans la citation où apparaissait l'expression de «fait linguistique », la division entre un système phonologique ou sémiologique " extérieur » et un système psychique « intérieur », partition refusée par Saussure au profit de la distinction entre son et son/idée ${ }^{18}$. On trouvera corrélativement un peu plus loin une opposition entre psychique et physique, remarquablement solidaire de la double problématique structurale et des rapports son/sens :

« La langue, on le voit, apparaît une construction à deux visages. Il y a le visage psychique, qui représente un morcellement savant du pensable, visant à pourvoir la pensée d'une faculté universelle d'expression. Et il y a en face, et inséparablement lié, le visage physique, constitué par des constructions de parole, dont l'idée s'attache inséparablement aux différentes parties du pensable, morcelé dans la langue d'une manière qui a pour objectif la possibilité universelle d'expression. » (Guillaume, 1989 : p. 14).

Il y a chez Guillaume, non division-combinaison, mais « morcellement » du pensable puis expression de la pensée ${ }^{19}$, et cette représentation n'a rien de théorique, mais elle est 
purement descriptive : comme dans la citation précédente, Guillaume affirme que « visage psychique » et "visage physique » sont «inséparablement liés », mais il ne nous dit ni comment ni pourquoi. La problématique descriptive - par opposition à théorisante apparaît de manière très nette dans la leçon du 20 février 1948. On y lit en effet :

« Dans la langue, au niveau de la langue, la liaison psychisme-parole est une liaison idéelle, selon laquelle le physique, qu'est en soi la parole, ne sort pas du psychique. Au niveau de la langue, la parole passée à l'état non physique, est un psychisme d'elle-même.

Dans le discours, au niveau du discours, les choses changent d'aspect, la liaison psychisme-parole devenant une liaison, idéelle encore, certes, par la conservation de ce qu'elle a été, mais selon laquelle le physique qu'est la parole en soi, se présente effectif, matérialisé, et donc, en ce qui le concerne, sorti de la condition psychique de départ. Au niveau du discours, la parole a pris corps, réalité : elle existe, physiquement, et n'est plus seulement psychisme d'elle-même. Ce psychisme d'elle-même qu'elle a été, elle ne le révoque pas, mais elle le réalise; elle lui confère une matérialité sensible, indispensable au langage, s'il ne reste pas intérieur. » (Guillaume, 1988 : p. 113-114).

Comme chez Saussure, la langue apparaît ici, par opposition à la parole, purement psychique. S'opposent cependant deux «liaisons psychisme-parole», donc deux rapports son/sens, au lieu que le caractère psychique de la langue saussurienne est corrélatif d'une définition de celle-ci comme division-combinaison, valant théorisation du rapport son/sens. Guillaume poursuit ainsi en parlant de «congruence », c'est-à-dire en constatant le « fait linguistique », à défaut de le théoriser :

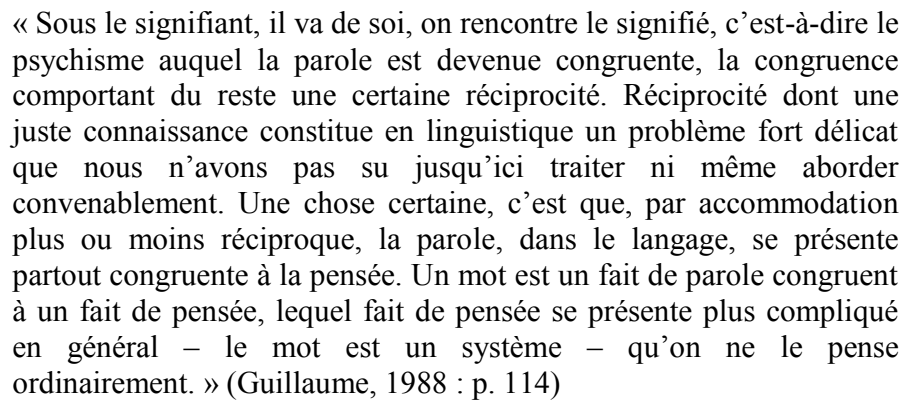

À cette double problématique structurale et des rapports son/sens, qui lie Guillaume au structuralisme européen, répond une problématique analytique ${ }^{20}$, qui entre néanmoins en tension - c'est là la spécificité de l'élaboration guillaumienne - avec une construction spéculative.

\section{Structuralisme et psychomécanique : entre analyse idiomologique et spéculation}

Les deux distinctions guillaumiennes entre langue et discours et entre langue et parole sont au service de l'analyse du donné idiomologique, et l'on retrouve ici la problématique analytique structuraliste. La congruence entre fait de pensée et fait de parole apparaît ainsi tout à la fois comme un objet et un principe d'analyse. Citons la leçon du 6 décembre 1947 :

« Ferdinand de Saussure oppose la parole à la langue, et démontre que l'étude de la parole est autre chose que celle de la langue. Cette vue est d'une profonde justesse, car la langue n'existe qu'en vertu d'une 
division savante du pensable en parties distinctes. À chacune des divisions correspond un fragment de parole qui en est le symbole. Mais il n'y a langue que pour autant qu'on considère la parole comme symbole de l'idée. Dès l'instant qu'on étudie la parole pour ellemême, indépendamment de sa valeur de symbole, on se désintéresse de la langue, et, selon Ferdinand de Saussure, et selon nous-même, on cesse de faire de la linguistique - la linguistique ayant pour objet la langue considérée en elle-même et pour elle-même. » (Guillaume, 1989 : p. 14).

Cette référence guillaumienne à Saussure est tout à fait remarquable, car elle témoigne de ce que Guillaume lit Saussure en y projetant sa propre démarche analytique : il n'est pas question de la parole comme phénomène, mais d'une étude de la parole à la seule lumière de la langue, et dès lors comme composant de la langue. On retrouve, mutatis mutandis, la démarche d'intégration du son à la langue qui est constitutive de la problématique phonologique ${ }^{21}$. L'idée, constitutive de la langue, est indépendante de la combinaison; elle servira ainsi de principe d'analyse ; Saussure oppose signe et son, Guillaume considère le son comme symbole de l'idée, d'avance linguistique. La convocation de la phrase finale du Cours de linguistique générale a dès lors une résonance presque hjelmslevienne. Citons également, dans la leçon précédente - 29 novembre :

«Un autre fait de grammaire générale, d'une importance sans égale, c'est que le langage et la langue supposent dans toutes leurs parties, et sur tous les plans, une suffisante congruence, une suffisante convenance d'un fait de parole et d'un fait de pensée. Autrement dit, pour qu'il y ait langage, et langue sous-jacente, il est nécessaire qu'un accord étroit soit intervenu entre la parole et la pensée. De sorte que le véritable objet de la linguistique, objet qui ne laisse rien échapper de cette science, est la connaissance aussi complète que possible de cet accord obligé. » (Guillaume, 1989 : p. 4-5).

On notera, dans cette affirmation, l'usage du passé : "soit intervenu ", qui relègue le « fait linguistique » au passé, et assigne corrélativement au linguiste un autre objet : le rapport son/sens, qui fournit un principe d'analyse du donné. On trouve d'ailleurs dans la leçon du 20 février 1948 une référence explicite à la phonologie :

«Il découle de là ${ }^{22}$ - et la science partielle, intérieure à la linguistique, qu'on appelle la phonologie n'a d'autre fondement - il découle de là que la parole-idée, faisant partie de la langue, est autre chose que la parole effective, qui en est une matérialisation. Et là nous allons retrouver une relation qui nous est connue à savoir que la multiplicité est du côté de l'effet, et l'unité relative du côté de la puissance. À la parole idéelle, conditionnellement une, s'oppose l'immense diversité de la parole effective, variable selon le sujet parlant, et aussi, pour un même sujet parlant, selon les circonstances de parole. » (Guillaume, $1988:$ p. 113)

Cette distinction entre puissance et effet, que Guillaume projette ici dans la distinction entre langue et parole, informe également, comme il est apparu plus haut ${ }^{23}$, la distinction langue/discours, qui constitue de même un cadre d'analyse des idiomes. À la langue et au discours correspondent effet deux plans constructeurs de deux types d'unités, requérant deux types d'analyse différents et déterminant la description des idiomes. On lit tout d'abord dans la leçon du 29 novembre 1946 :

«Cette distinction de la langue et du discours, dont il importe, avant d'entreprendre quelque étude de linguistique que ce soit, de se représenter l'importance, est un fait de grammaire générale, duquel 
dépendront ensuite la totalité des faits de grammaire particulière. » (Guillaume, 1989 : p. 3)

Suit l'exemple de l'article ${ }^{24}$, qui fait clairement apparaître que la distinction entre langue et discours constitue un cadre d'analyse des idiomes. Guillaume met ensuite en évidence un «fait essentiel de grammaire générale » (Guillaume, 1989:p. 6) : « le discours est constructeur d'unités d'effet, éphémères en principe, et la langue constructrice d'unités de puissance, permanentes, en principe, dans l'esprit, exemptes de momentanéité » (Guillaume, 1989 : p. 6). On lit alors :

« D’une manière générale, un linguiste attentif doit avoir un sentiment clair de ce qui, dans un idiome qu'il étudie, ressortit au plan de puissance et au plan de l'effet. D'un idiome à l'autre, les choses, à cet égard, peuvent différer assez considérablement. » (Guillaume, 1989: p. 6$)^{25}$.

Il s'agit bien d'élaborer une représentation permettant l'analyse la plus adéquate possible des idiomes. Il s'agit en outre de déployer deux types d'analyse distincts :

«Une chose est l'économie qui régit les constructions du discours, et autre chose celle qui régit la langue, les constructions de langue. Il suit de là qu'il ne peut être question d'appliquer à la langue l'analyse qui convient au discours. La langue relève d'une analyse adéquate à son économie; et cette analyse, nous aurons l'occasion de le voir, est quelque chose de tout à fait original et particulier. » (Guillaume, 1989 : p. 13$)^{26}$.

Comme Guillaume l'explique dans les lignes qui précèdent ${ }^{27}$, puis de nouveau plus $\operatorname{loin}^{28}$, les visées de la langue et du discours sont en effet radicalement différentes l'une de l'autre : la première est universelle, alors que la seconde est particulière. C'est pourquoi, comme Guillaume l'affirmait à propos de la distinction entre langue et parole ${ }^{29}$, l'unité est du côté de la langue, la multiplicité étant l'apanage du discours :

« Partout dans le langage, et c'est là, de nouveau, un fait de grammaire générale, on rencontre le principe de l'unité de la condition de langue en face de la multiplicité des conséquences de discours, à partir d'une même condition. [...]

Une visée constructive constante de la langue est de ramener à un nombre restreint de conditions transcendantes, et pour autant simples - la transcendance assure la simplicité - la multiplicité infinie des visées du discours, qui sont souvent d'une subtilité extrême.» (Guillaume, 1989 : p. 15-16) ${ }^{30}$.

On est proche, ici, mutatis mutandis, de la construction jakobsonienne en termes d'invariance dans la variation, à ceci près que s'il s'agit, comme chez les structuralistes, de construire la langue pour rendre compte de la parole, cette construction implique une ordonnance de la langue et du discours, ainsi qu'une dualité d'analyse, langue et discours étant tous deux le produit d'une construction: «[...] de même que la langue se construit dans la sous-jacence du plan de puissance, le discours se construit dans la sous-jacence du plan de l'effet. La symétrie est remarquable.»(Guillaume, 1989: p. 11). La langue demeure néanmoins le premier objet du linguiste, et en tout cas la condition d'une analyse véritablement linguistique. On lit ainsi dans la leçon du 6 décembre 1946 :

«Il est impossible d'étudier le discours sans du même coup tenir compte de la langue. De là vient que quiconque étudie le discours fait par cela même de la linguistique. Mais il ne faut pas perdre de vue que le vrai linguiste, le linguiste complet, est celui qui, avant de s'intéresser au discours, qui est de l'ordre de la conséquence, s'est intéressé profondément à la langue qui est, elle, de l'ordre de la 
condition. Or si d'une manière générale on s'intéresse de toute part beaucoup au discours, aux faits de discours, à leurs nuances, peu nombreux sont les esprits qui réservent le principal de leur attention à la langue.

Le peu d'attention suivie que l'on a donnée à la langue, aux faits de langue, considérés dans leur plan propre, le plan de puissance, est l'une des causes qui ont le plus retardé l'avancement de la linguistique. »(Guillaume, 1989 : p. 14-15).

L'élaboration guillaumienne de la distinction saussurienne entre langue et parole s'inscrit ainsi, comme celle des structuralistes, dans une problématique analytique. C'est là un enjeu du dédoublement guillaumien de la distinction saussurienne : la distinction langue/discours construit le cadre de l'analyse idiomologique, cependant que la distinction langue/parole constitue la langue (le rapport son/sens) comme principe d'analyse. La structure temporelle de la distinction entre langue et discours est cependant solidaire d'une visée théorisante, dans le cadre de la caractérisation de l'acte de langage. C'est là, en effet, l'enjeu du partage d'objet que je me suis efforcée de mettre en évidence plus haut. Comme nous l'avons vu, dans la leçon du 6 décembre 1946, Guillaume caractérise l'acte de langage comme ce qui permet de passer de la langue au discours ${ }^{31}$. L'année suivante, la définition du langage comme intégrale de la langue et de la parole (du discours) implique de même l'établissement par ce dernier d'un lien entre les deux :
«[...] la visée de puissance et la visée d'effet sont hétérogènes par le contenu opératif, nonobstant l'accord de caractère pragmatique qui s'établit entre elles, en vertu du langage, lequel, totalisant les deux visées, il en est l'intégrale, utilise les résultats de la première à l'obtention des fins que poursuit la seconde. »(Guillaume, 1988: p. 117).

Guillaume reprend à son compte la définition traditionnelle de la langue comme instrument de communication, dont témoigne l'équivalence entre langage et acte de langage, et qui apparaît de manière tout à fait explicite en particulier dans les premières leçons de l'année 1946-1947. Il est en effet question, tout au long de ces leçons, d'expression de la pensée. Citons notamment :

«La langue est ce qui, dans la vue de permettre plus aisément l'expression de la pensée, s'est déterminé, construit préalablement, au titre de moyens permanents, sur le plan de puissance. » (Guillaume, 1989 : p. 9$)^{32}$.

C'est alors la langue qui apparaît comme un outil d'expression de la pensée, mais cette caractérisation intervient sur le fond d'une appréhension du langage comme acte de langage. Dans cette perspective, la langue apparaît comme une modalité du langage, d'avance défini comme communication. On lit en particulier dans la leçon du 6 décembre 1946 :

« L'acte de langage est un acte qui a une économie interne, et doit être étudié du point de vue de cette économie. L'acte de langage est d'autant plus léger, plus aisé, plus facile, qu'il a derrière soi, dans la vue de se donner aisance et puissance, une langue mieux construite. Dans le cas d'une langue insuffisamment construite, ou mal construite, l'acte de langage est un acte difficile, de résultat incertain, et manquant souvent de moyens.

Dans l'hypothèse d'une absence de langue, préalablement construite dans la pensée du sujet parlant, l'acte de langage devient un acte si lourd, si chargé qu'il constitue une impossibilité. Pour son succès, il est en effet alors nécessaire d'improviser, de créer, dans le 
moment du besoin, des moyens d'expression. C'est là quelque chose qui passe la capacité humaine.

Un fait de grammaire générale à ajouter à ceux dont j'ai déjà fait mention, c'est que, depuis les origines, l'acte de langage a tendu incessamment à s'alléger en déférant à la langue, créée au-dessous de lui, tout ce qui était pour lui une charge excessive. La charge pleine de l'acte de langage, c'est de n'avoir pas de langue au-dessous de lui. L'allègement de l'acte de langage, c'est d'avoir < au-dessous $>$ de lui une langue où tout est déjà préparé savamment, et inconsciemment, de ce qu'il aura à entreprendre. La langue est un allègement de l'acte de langage. » (Guillaume, 1989 : p. 12).

Si la distinction saussurienne entre langage et langue est délimitation d'un objet spécifiquement linguistique, son homonyme guillaumienne constitue le cadre d'une pseudo-étiologie - «pseudo »-étiologie, puisque cette étiologie se fonde sur un donné préalable : celui de la définition commune du langage - de la langue comme produit de la pensée, aux fins de l'acte de langage. C'est là le mécanisme du discours dont il a été question plus haut, et qui vaut également étiologie - c'est-à-dire pseudo-étiologie - de la langue. Citons notamment, dans la leçon du 20 février 1948 :

«Dans la langue, au niveau de la langue, la congruence fait de parole/fait de pensée ressortit à la visée constructive de la langue. Dans le discours, la congruence fait de parole/fait de pensée - qui est dans le langage, qu'on veuille bien en faire la remarque, une constante - ressortit à la visée constructive du discours. Or les deux visées se lient l'une à l'autre selon une relation de successivité, d'un caractère original. La visée de puissance construit des unités de puissance et des mécanismes de puissance, dont la réunion constitue la langue. Et la visée d'effet saisit au résultat ce que la visée de puissance a édifié, et elle s'en sert : elle se sert des résultats acquis en visée de puissance, pour construire, dans le momentané, le discours. Cette liaison, en vue de l'utile, des deux visées, dont la seconde se sert selon ses fins propres de ce que la première a produit, selon ses fins propres aussi, ne signifie pas que, par leur contenu opératif, les deux visées soient assujetties à la moindre condition d'identité. De fait, et l'expérience le montre, le contenu opératif de la visée de puissance est d'une autre essence que celui de la visée d'effet.

La visée de puissance fait appel, selon sa nature propre, à des opérations de pensée en petit nombre, toujours les mêmes, qui sont celles-là même auxquelles la pensée doit sa puissance. Il suit de là, et la valeur du principe n'échappera à personne - il suit de là que les opérations présidant à la structure de la langue sont exactement celles qui apportent à la pensée sa puissance constructive. » (Guillaume, $1988:$ p. 114-115)

La langue guillaumienne se trouve ainsi définie, dans ce cadre de la définition du langage comme outil de communication (acte de langage), comme produit de la pensée. Cette définition, qui suppose l'acceptation de la définition commune du langage, apparaît pour cette raison purement spéculative. En témoigne la valorisation de la langue comme constructrice d'universalité, très lisible dans la leçon du 6 décembre 1946, où Guillaume affirme que la langue, « allègement de l'acte de langage » (Guillaume, 1989 : p. 12), « devient, portée à un haut degré de perfection, une prévision universelle de tout ce qui peut être exprimé : une prévision universelle de l'exprimable» (Guillaume, 1989: p. 12) et que tandis que le discours est de «visée particulière - même étroitement particulière » (Guillaume, 1989: p. 12), la langue a prévu tous les effets de discours, «non pas un à un, séparément, individuellement, mais d'une manière transcendante, selon une économie supérieure qui permet de les obtenir tous, si divers soient-ils et si grand qu'en soit le nombre, à partir de moyens en nombre limité formant 
système » (Guillaume, 1989: p. 13). Cette valorisation, d'autant plus remarquable que se confondent ou se conjoignent supériorité de la langue et efficience de la construction du linguiste comme outil d'analyse idiomologique - à travers la notion de prévision -, apparaît également à travers la récurrence de la notion de profondeur qui vient doubler l'ordonnance temporelle de la langue et du discours : « la parole est une chose au niveau profond de la langue, et autre chose au niveau superficiel du discours » (Guillaume, $1988:$ p. 114), lit-on dans la leçon du 20 février 1948. Cette notion est théoriquement inconsistante, comme il apparaît en particulier dans ce passage de la leçon du 6 décembre 1946 :

«Ce n'est que sur ce plan - le plan de puissance - que l'on rencontre la langue. La construction de la langue est une opération de pensée qui aboutit à ce plan, qui se termine à ce plan. Or ce plan est dans la pensée un plan profond. La construction de la langue est donc une opération profonde de la pensée, puisque sur le plan de puissance, qui est lui-même un plan profond, elle se présente terminée. » (Guillaume, 1989 : p. 9$)^{33}$.

La proposition suivante, extraite de la leçon du 26 novembre 1948, témoigne nettement de ce qu'elle vient en lieu et place de toute théorisation - dont elle voile la nécessité :

«La parole de puissance, qui n'est qu'une idée de parole, est celle liée au mot avant même que nous en fassions usage. La liaison est d'ordre psychique : elle est un fait de pensée profond. » (Guillaume, 1973 : p. 19).

C'est en effet alors l'union du son et du sens, que, comme nous l'avons vu plus haut, Guillaume se contente de constater, qui s'y trouve décrite comme «fait de pensée profond». La valorisation apparaît enfin ${ }^{34}$ dans l'idéologie indoeuropéencentriste de Guillaume, qui parle volontiers de « nos langues évoluées » (Guillaume, 1989 : p. 11).

Il apparaît ainsi que la lecture guillaumienne de la distinction saussurienne entre langue et parole se singularise par son double caractère analytique et spéculatif. La leçon du 13 décembre 1946 se conclut sur l'évocation du «temps constructif» (Guillaume, 1989: p. 18), que Guillaume appelle ailleurs «temps opératif », et qui apparaît ainsi comme un pendant analytique et spéculatif de la définition saussurienne de la langue comme fonctionnement :

« On ne saisit un système, un état de système, que par reconstruction mentale instantanée. C'est là une vérité générale applicable en tous domaines. Cette reconstruction mentale s'identifie avec le système même, qui n'existe comme tel que si elle existe. » (Guillaume, 1989 : p. 19).

Cette notion est au centre de la neurolinguistique élaborée par Gisèle Gelbert, et plus généralement, l'élaboration guillaumienne d'une représentation du mécanisme du discours est en parfaite consonance avec les réflexions actuelles de la linguistique cognitive, paradigme dominant dans les neurosciences. Il importe d'autant plus, à cet égard, de prendre la mesure du caractère spéculatif d'une telle représentation, dont, comme je me suis efforcée de le montrer, la construction est avant tout déterminée par le double obstacle épistémologique de l'idiome et de l'unité du langage. Le langage, qui devrait être objet de théorisation, fonctionne ainsi tout à l'inverse comme cadre de théorisation. Comme tel, il ne saurait être qu'objet de spéculations, fussent-elles, lorsqu'il s'agit de neurobiologie, «scientifiques». Une neurobiologie saussurienne permettrait peut-être en revanche de redonner à l'adjectif scientifique sa vigueur étymologique, au lieu que son usage conduise à effacer toute différence entre l'héritier de facio et le suffixe -iste. 


\section{Références bibliographiques}

Decimo, M. (1994), « Saussure à Paris ». Cahiers Ferdinand de Saussure, 48, p. 75-90.

Dedet, A. (1995). «La référence à Saussure chez G. Guillaume ». Arrivé, M. \& Normand, C. (dir.), Saussure aujourd'hui, numéro spécial de LINX, p. 461-467. Université Paris $\mathrm{X}$-Nanterre : Centre de recherches linguistiques.

Godel, R. (1957). Les sources manuscrites $d u$ Cours de linguistique générale de F. de Saussure. Genève : Droz.

Guillaume, G. (1973). Leçons de linguistique de Gustave Guillaume 1948-1949 C. Grammaire particulière du français et grammaire générale IV. Paris : Klincksieck / Québec : Presses de l'université Laval.

Guillaume, G. (1988). Leçons de linguistique de Gustave Guillaume 1947-1948 C. Grammaire particulière du français et grammaire générale III. Lille: Presses universitaires du Septentrion.

Guillaume, G. (1989). Leçons de linguistique de Gustave Guillaume 1946-1947 C. Grammaire particulière $d u$ français et grammaire générale II. Lille: Presses universitaires du Septentrion.

Guillaume, G. (2000). Leçons de linguistique de Gustave Guillaume. Série A. 1949-1950. Structure sémiologique et structure psychique de la langue française II. Paris: Klincksieck / Québec : Presses de l'université Laval.

Jakobson, R. (1988). Selected Writings VIII. Berlin, New York, Amsterdam : Mouton Publishers.

Joly, A. \& Roulland, D. (1981). «Pour une approche psychomécanique de l'énonciation ». Joly, A. \& Hirtle, W. H. (éds). Langage et psychomécanique du langage, Études dédiées à Roch Valin, p. 537-581. Québec : Presse de l'université Laval / Lille : Presses universitaires de Lille.

Joly, A. (1987). Essais de systématique énonciative. Lille : Presses universitaires de Lille.

Saussure, F. (de) (1972 [1995]). Cours de linguistique générale. Paris : Payot.

Saussure, F. (de) (1996). Premier Cours de linguistique générale (1907), d'après les cahiers d'Albert Riedlinger. Oxford, New York, Séoul, Tokyo : Pergamon.

Saussure, F. (de) (1997). Deuxième Cours de linguistique générale (1908-1909), d'après les cahiers d'Albert Riedlinger et Charles Patois. Oxford, New York, Tokyo : Pergamon.

Saussure, F. (de) (2002). Écrits de linguistique générale. Paris : Gallimard ${ }^{35}$.

Saussure, F. (de) \& Constantin, É. (2005). «Ferdinand de Saussure : Notes préparatoires pour le cours de linguistique générale 1910-1911, Emile Constantin: Linguistique générale. Cours de M. le professeur de Saussure 1910-1911 ». Cahiers Ferdinand de Saussure, 58, p. 83-289.

Toutain, A.-G. (2014). La rupture saussurienne. L'espace du langage. Louvain-la-neuve : Academia-Bruylant.

Toutain, A.-G. (2015). La problématique phonologique. Du structuralisme européen comme idéologie scientifique. Paris : Classiques Garnier.

Toutain, A.-G. (2016). Entre langues et logos. Une analyse épistémologique de la linguistique benvenistienne. Berlin : De Gruyter. 
Valette, M. (2003). «Énonciation et cognition : deux termes in absentia pour des notions omniprésentes dans l'œuvre de Guillaume. » Le Français moderne, LXXXI-1, p .6-25.

Valette, M. (2006). Linguistiques énonciatives et cognitives françaises. Gustave Guillaume, Bernard Pottier, Maurice Toussaint, Antoine Culioli. Paris : Honoré Champion.

${ }^{1}$ Voir notamment Toutain (2015).

${ }^{2}$ C'est-à-dire comme une " chose », au sens usuel du terme, une entité existant comme telle, fût-elle dotée d'une matérialité singulière.

${ }^{3}$ Voir Toutain (2014).

${ }^{4}$ Voir Joly \& Roulland (1981) et Joly (1987), ainsi que les travaux de Valette en référence dans la note suivante.

${ }^{5}$ Voir Valette (2003) et Valette (2006).

${ }^{6}$ Dualité qui, bien que sa forme soit nettement différente, n'est pas sans évoquer la singularité de l'élaboration benvenistienne au sein du paradigme structuraliste. Voir Toutain (2016).

${ }^{7}$ Voir également Saussure \& Constantin (2005) : p. 88.

${ }^{8}$ Titre du premier paragraphe du quatrième chapitre de la deuxième partie du Cours de linguistique générale.

${ }^{9}$ Les termes de parole et de discours apparaissent certes ponctuellement dans des notes autographes antérieures, mais il y a loin de ces occurrences à l'élaboration de la distinction langue/parole. Voir - dans l'ordre chronologique - Saussure (2002) : p. 159, p. 61-62, p. 105 et 117-118, ainsi que la dite « Note sur le discours », non datée, et Saussure (2002) : p. 95 et 130 (deux autres notes non datées). Le « Rapport sur la création d'une chaire de stylistique » (19121913), où apparaît la distinction langue/parole, est quant à lui postérieur au troisième cours.

${ }^{10}$ Voir la lettre à Gaston Paris du 30 décembre 1891 : Decimo (1994) : p. 78-79.

11 Voir «De l'essence double du langage », ainsi que les « Notes pour un livre sur la linguistique générale », « Statut et motus » et la note « Morphologie », in Saussure (2002).

${ }^{12}$ Un apport majeur de la théorisation saussurienne de la langue est ainsi la distinction entre langue, langage, et idiome. Pour cette distinction, voir par exemple Toutain (2014).

${ }^{13}$ Il s'agit d'une série de leçons de quatre années qui se clôt en 1949. J'analyserai, à titre d'échantillon, les leçons de 1946-1947 et celles de 1947-1948. Si l'élaboration se modifie et se complexifie au fil des leçons et des années, la problématique - qui fait l'objet de notre analyse - demeure inchangée.

${ }^{14}$ Ce que Guillaume affirme explicitement un peu plus loin : «la parole - laquelle, dans le langage représente le signifiant» (Guillaume, 1988: p. 114). Voir aussi Guillaume (1989) : p. 13 .

15 On peut bien entendu discuter de la pertinence des termes choisis par Guillaume, discours au lieu de parole, la parole étant redéfinie comme le pôle du signifiant par opposition à la pensée. Là, néanmoins n'est pas l'important, dans la mesure où ce sont les contenus des concepts, et non leurs noms, qui sont décisifs. Cette contribution s'efforce ainsi de mettre en évidence les enjeux, en termes de problématique, de la réélaboration guillaumienne de la distinction saussurienne.

${ }_{16}$ « J'ai, par position, c'est-à-dire en suivant les méthodes de ce qu'il m'arrive d'appeler la linguistique de position, défini ce qu'est la langue, d'une part, le discours, d'autre part ; et entre les deux, pour passer de la première au second, j'ai inscrit l'acte de langage. » (Guillaume, 1989 : p. 13).

${ }^{17}$ Voir en particulier «La théorie saussurienne en rétrospection» (1942), in Jakobson (1988).

${ }^{18}$ On lit ainsi dans « De l'essence double du langage »: « Le dualisme profond qui partage le langage ne réside pas dans le dualisme du son et de l'idée, du phénomène vocal et du phénomène mental; c'est là la façon facile et pernicieuse de le concevoir. Ce dualisme réside dans la dualité du phénomène vocal COMME TEL, et du phénomène vocal COMME SIGNE - du fait 
physique, (objectif) et du fait physico-mental (subjectif), nullement du fait "physique" du son par opposition au fait "mental" de la signification. Il y a un premier domaine, intérieur, psychique, où existe le signe autant que la signification, l'un indissolublement lié à l'autre ; il y en a un second, extérieur, où n'existe plus que le "signe"; mais à cet instant le signe réduit à une succession d'ondes sonores ne mérite pour nous que le nom de figure vocale.» (Saussure, 2002 : p. 20-21).

19 Il sera d'ailleurs question ultérieurement, respectivement, de représentation et d'expression. Voir par exemple Guillaume (1973) et Guillaume (2000).

${ }^{20}$ Le terme analytique renvoie à une démarche d'analyse du donné, en lieu et place de toute théorisation. Il s'agit ici d'analyse de la parole (ou du discours) dans le cadre du postulat et de la construction du système linguistique.

${ }^{21}$ Voir Toutain (2015).

${ }^{22}$ Guillaume vient d'énoncer la distinction entre parole effective et parole virtuelle ; voir Guillaume (1988) : p. 112-113 supra.

${ }^{23}$ Voir Guillaume (1989) : p. 9, cité supra, ainsi que Guillaume (1988) : p. 110.

${ }^{24}$ Voir Guillaume (1989) : p. 3-4.

${ }^{25}$ Voir ensuite Guillaume (1989) : p. 6-7.

${ }^{26}$ Voir ensuite Guillaume (1989) : p. 14-15, 17 et 18.

${ }^{27}$ Voir Guillaume (1989) : p. 12-13.

${ }^{28}$ Voir Guillaume (1989) : p. 15-16 puis 17-18.

${ }^{29}$ Voir Guillaume (1988) : p. 113, ci-dessus.

${ }^{30}$ L'affirmation est reprise à la page suivante (Guillaume, 1989 : p. 17).

${ }^{31}$ Voir Guillaume (1989) : p. 13, cité supra. On lisait de même deux pages avant : «La construction du discours à partir de la langue est un acte de langage. L'acte de langage consiste à passer de la langue au discours. » (Guillaume, 1989 : p. 11).

32 On lit de même ensuite : «C'est à la langue construite en lui, et dont il a la possession permanente, que le sujet parlant s'adresse lorsqu'il veut, ou lorsqu'il lui faut exprimer sa pensée. Le succès de l'expression de sa pensée dépend étroitement de ce qu'est en lui la langue à laquelle le sujet parlant fait appel. » (Guillaume, 1989 : p. 10), puis de nouveau : «Le sujet parlant qui, pour extérioriser sa pensée, s'adresse à la langue, se propose, avec les éléments qu'elle va lui fournir, de construire un discours limité, duquel il attend certains effets auxquels sera sensible - du moins il le présume - le sujet écoutant. » (Guillaume, 1989 : p. 11).

${ }^{33}$ Voir également, notamment, Guillaume (1989) : p. 8, 13, 17 et 19.

${ }^{34}$ Notons par ailleurs l'insistance, dans les premières leçons de l'année 1946-1947, sur le caractère « secret » des opérations du plan de puissance : «la construction du mot, opérée plus secrètement sur le plan de puissance» (Guillaume, 1989: p. 6), «Toutes les opérations constructives dont le résultat est la langue - qui, donc, se terminent au plan de puissance - sont à un haut degré des opérations secrètes, qui ne peuvent être décelées que par une analyse spéciale, pourvue de moyens appropriés. » (Guillaume, 1989 : p. 10).

${ }^{35}$ Toutes les citations ont été corrigées sur les manuscrits, et le texte peut donc différer de celui de cette publication. 